INRA Prod. Anim., 2007, 20 (4), 327-336

\title{
Relations entre pratiques de finition et performances à l'abattage de vaches de réforme Simmental Française : intérêt du caractère mixte de la race
}

\author{
C. GAILLARD ${ }^{1}$, S. INGRAND', J. BLANQUET ${ }^{3}$, C. DEVEVEY', M. ROUX'. \\ ${ }^{1}$ ENESAD, UR Systèmes d'élevage et Qualité des Animaux et des Viandes, 26 Bd Petitjean' F-21079 Dijon, France \\ ${ }^{2}$ INRA, ENGREF, CEMAGREF, ENITA, UMR1273 Mutations des Activités, des Espaces et des Formes d'Organisation \\ dans les Territoires Ruraux, F-63122 Saint-Genès Champanelle, France \\ ${ }^{3}$ ENESAD, Laboratoire de Mathématiques Appliquées à l'Informatique et aux Statistiques, 26 Bd Petitjean' \\ F-21079 Dijon, France \\ Courriel : c.gaillard@enesad.fr
}

La race Simmental Française se distingue des races spécialisées par le maintien d'un équilibre entre aptitudes laitières et bouchères. Alors que les performances laitières, évaluées par le contrôle laitier sont bien connues, la production de viande est peu étudiée. Les caractéristiques bouchères des vaches de réforme, mises en relation avec les pratiques d'engraissement observées dans le berceau de la race, révèlent une très grande variabilité. Celle-ci est expliquée par différents degrés dans l'expression du caractère mixte de la race, selon les objectifs des éleveurs, les ressources dont ils disposent et les contraintes auxquelles ils ont à faire face.

\begin{abstract}
Parmi les races bovines laitières françaises, plusieurs races traites se distinguent par leur potentiel de production de viande. Parmi elles, la Simmental est traditionnellement implantée dans 1'Est de la France avec un berceau de race dans le département de Haute-Marne. Comptant parmi les premières races bovines françaises avec un effectif d'environ 500000 vaches dans les années 1950, elle a connu par la suite un déclin intense et continu jusque dans les années 1980 (Gaillard 1995). Depuis cette date, tout en étant très minoritaire puisqu'elle représente alors $0,9 \%$ des effectifs laitiers contrôlés, elle suscite quelques pôles de développement d'abord dans le Sud du Massif Central (pôle d'expansion dans l'Aveyron qui représente actuellement un quart de l'effectif contrôlé, FCL

tion du potentiel laitier tout en maintenant les aptitudes bouchères. Pour cela, les responsables de la sélection s'appuient d'une part sur le dispositif national de sélection laitière permis par le Contrôle Laitier des troupeaux et d'autre part sur la mobilisation de géniteurs étrangers proches, notamment de race Fleckvieh allemande et autrichienne. En ce qui concerne l'aptitude viande, l'utilisation des index «aptitudes bouchères» de ces taureaux étrangers ainsi qu'une appréciation morphologique subjective des candidats à la sélection, juste avant leur mise en testage, pallient l'absence de dispositif spécifique à la sélection bouchère. Un tel dispositif ne peut pas être envisagé en France, en raison de l'effectif limité de la population qui s'élève à 13767 vaches contrôlées (FCL 2004).
\end{abstract} 2004), puis plus récemment et de manière sporadique dans diverses régions françaises (zones herbagères de Lorraine ou de l'Ouest : Morbihan, Ille-et-Vilaine, Vendée). L'objectif recherché par ces éleveurs quittant généralement un cheptel de race Prim'Holstein, se situe dans l'équilibre lait-viande ainsi que dans l'équilibre taux butyreux-taux protéique du lait produit, caractérisant les animaux de race Simmental (Audiot et al 2004). De fait, dans cette race, le programme de sélection mis en œuvre vise l'améliora-
L'infusion de génétique Fleckvieh semble satisfaire les éleveurs utilisateurs de Simmental Française. Mais comment caractériser l'équilibre établi entre les productions de lait et de viande ? Audelà des aptitudes laitières bien évaluées par le Contrôle Laitier, peut-on préciser les aptitudes bouchères actuelles du cheptel Simmental ? Comment relier l'expression de ces aptitudes avec les pratiques de conduite des éleveurs ? Sont-elles spécifiques en raison du caractère mixte de la race ?
Pour répondre à ces questions, l'analyse a consisté à relier des données issues de fichiers d'abattoir à des données issues d'enquêtes réalisées dans des élevages permettant d'appréhender «en amont» les pratiques d'élevage correspondantes (Landais 1987) puis d'identifier des types d'élevage selon leurs systèmes de pratiques (Cristofini 1978), afin de montrer qu'on peut les associer à différents degrés d'expression de la mixité. Pour appréhender les performances bouchères et les modes de production en amont, nous avons donc construit un dispositif en trois étapes, partant de la collecte de données en abattoir (1 ère étape), cherchant ensuite à repérer des profils de performances bouchères (2ème étape), puis visant à caractériser la diversité des modes de production selon ces profils, par l'étude des pratiques d'élevage (3ème étape).

\section{1 / Méthodologie}

\section{1 / Les vaches de réforme comme support d'analyse}

Dans le cas de la race Simmental, les vaches de réforme, vendues pour la viande à l'issue de leur carrière laitière, sont considérées par les éleveurs comme l'ar- 
chétype de l'aptitude de mixité, en référence à la conduite d'un troupeau de race laitière spécialisée. En outre, par comparaison aux mâles, également supports de production de viande, il nous semble que les vaches, étant donné leur fonction et leur longévité dans le troupeau, laissent davantage d'empreintes dans la mémoire de l'éleveur, mobilisables au cours d'enquêtes en élevage.

\section{2 / Performances bouchères à partir des données d'abattage}

\section{Collecte des données}

Des données d'abattage ont été collectées dans neuf abattoirs de l'Est de la France, sur une période allant du 1 er janvier 2000 au 30 juin 2002. Le choix de ces abattoirs est justifié par une présence significative d'animaux Simmental (code $35^{1}$ ). Pour chaque animal, les données figurant sur le ticket de pesée ont été relevées : numéro d'identification de l'animal, date d'abattage, poids de carcasse froide, classement commercial de la conformation selon la grille communautaire EUROP (règlement CEE n ${ }^{\circ} 2930 / 81$ de la commission du 12 octobre 1981) et état d'engraissement de la carcasse.

Une connexion avec le fichier national d'identification (INRA-CTIG de Jouy-en-Josas) a permis en outre d'obtenir la date de naissance, le numéro d'élevage dont provient l'animal, et dans la mesure du possible, le numéro d'identification de son père. Toutefois, $15 \%$ des vaches n'ont pas pu être associées à un numéro d'élevage et ont donc été exclues de l'analyse.

La base contient 7957 vaches de réforme issues de 1013 élevages. Les

Figure 1. Différentes modalités des variables d'abattage.

Age (ans) : $<4,4-5,6-9,>=10$
Poids carcasse $(\mathrm{kg}):<320,320-339,340-369,370-399,>=400$
Conformation de la carcasse (classes) : EUR, O+, O=, O-P
Saison d'abattage : décembre à février, mars à mai, juin à août, septembre à novembre

performances des vaches à l'abattage ont été décrites à l'aide de trois variables : âge, poids de carcasse froide et conformation de la carcasse. La note d'état a été mobilisée pour ses valeurs 1 et 2, témoignant d'une finition incomplète. En revanche, la valeur 3, moins fiable, n'a pas été prise en compte.

\section{Construction de profils de performances}

L'analyse a porté sur des vaches de réforme issues de trois départements du berceau de la race (Côte-d'Or, HauteMarne et Jura), de manière à disposer d'un échantillon à la fois suffisamment vaste et proche géographiquement pour la réalisation ultérieure d'enquêtes auprès des éleveurs. L'échantillon comprend ainsi 2743 vaches provenant de 122 élevages. Nous avons cherché à définir des «profils de performances», par une analyse factorielle des correspondances (AFC, logiciel Winstat) sur les variables âge, poids et classement commercial de la carcasse, les individus étant les élevages. Chaque variable est répartie en classes, en fonction de la distribution des individus et en veillant à distinguer certaines catégories d'âge, notamment les primipares et les vaches les plus âgées (figure 1).

\section{3 / Recueil et analyse des pra- tiques d'élevage}

A partir des profils de performances issus de l'analyse précédente, un échantillonnage stratifié de vingt-cinq élevages a été réalisé en choisissant des exploitations situées graphiquement au centre des profils auxquels elles appartiennent ainsi qu'en nous assurant de la présence d'une production de viande significative (effectif minimum de cinq animaux commercialisés par catégorie, mâle ou vache de réforme). L'origine géographique départementale des exploitations ne constitue pas un facteur d'échantillonnage.

Les élevages retenus ont fait l'objet d'une seule enquête, sous la forme d'un entretien semi-directif, conçu autour de trois champs d'investigation :

- la conduite alimentaire du troupeau laitier : composition de la ration de base (importance respective de l'ensilage de maïs, du foin), estimation du niveau annuel de complémentation concentrée et du niveau de productivité laitière,

- la production de viande abordée à travers les pratiques de réforme et de finition des vaches : modalités de la décision de finition, pratiques mises en œuvre (tarissement, conditions de finition, composition de la ration de base, niveau de complémentation en céréales) et évaluation de la durée de finition (figure 2),

- la gestion des accouplements : recensement des critères retenus par l'éleveur dans le choix des taureaux et

Figure 2. Différentes modalités des variables liées aux pratiques d'élevage.

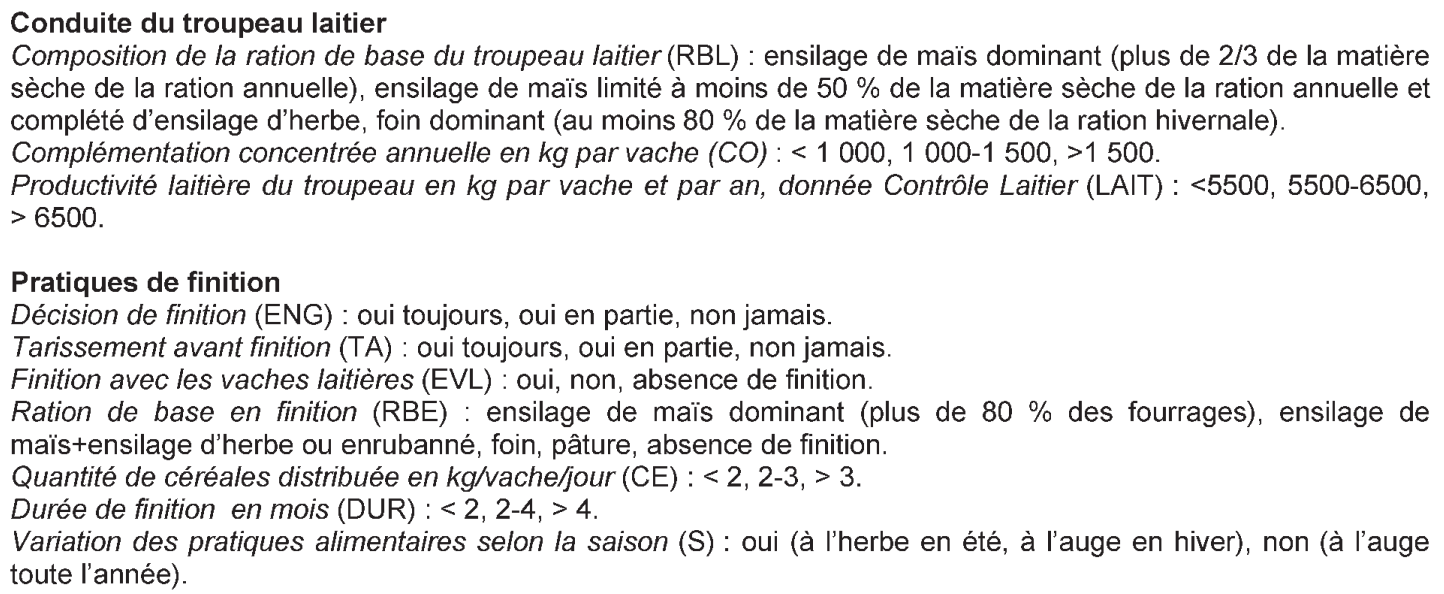

${ }^{1}$ La mention du code numérique racial (35 pour la race Simmental Française) est obligatoire sur le ticket de pesée. 
la sélection des femelles, de leur ordre d'importance (production laitière, critères fonctionnels (taux cellulaire, fertilité, facilité de traite) et morphologie bouchère) ainsi que du seuil laitier de tri des primipares lors des deux premiers contrôles laitiers ou sur la première lactation.

Dans un premier temps, l'ensemble des variables collectées a fait l'objet d'une Analyse Factorielle des Correspondances Multiple (AFCM) et d'une Classification Ascendante Hiérarchique (CAH) sur la base des coordonnées des individus sur les axes les plus significatifs (logiciel Winstat).

Dans un second temps, nous avons cherché à mettre en lien les pratiques d'élevage et les performances bouchères en croisant les profils de performances à l'abattage et les types d'élevages identifiés selon leurs pratiques.

\section{2 / Résultats}

\section{1 / Quatre profils de perfor- mances bouchères des vaches de réforme}

Sur la période d'étude retenue correspondant à un peu plus de deux campagnes laitières, l'effectif moyen de vaches réformées par élevage est de 7 avec toutefois une variabilité très importante qui résulte en partie de la diversité des tailles d'élevage mais également de la composition raciale du troupeau (présence d'autres races que Simmental dans le même troupeau).

L'âge moyen à l'abattage est de 6 ans et 11 mois avec un écart-type de 2 ans et 11 mois. La majorité des vaches $(80 \%)$ est abattue à moins de 10 ans dont $31 \%$ à moins de 5 ans. Le poids moyen des carcasses est de $334 \pm 48,7$ $\mathrm{kg}$ tandis que la conformation moyenne se situe entre $\mathrm{O}=$ et $\mathrm{O}+$ (variant entre O- et R-).

La liaison entre poids de carcasse et classement commercial (figure 3) indique que sur l'ensemble de la gamme de poids, la répartition est équilibrée entre les classes $\mathrm{O}$ et $\mathrm{R}$. La classe $\mathrm{P}$ n'est observée que pour des carcasses de poids inférieur à $300 \mathrm{~kg}$, tandis que la classe $\mathrm{U}$ concerne des carcasses de plus de $400 \mathrm{~kg}$. Les trois premiers axes de l'AFC réalisée sur les 122 élevages de la zone berceau, expliquent $58 \%$ de la variabilité totale des performances bouchères. Les élevages apparaissent ordonnés dans le plan fac-

Figure 3. Répartition du classement EUROPA des carcasses de vaches de réforme vendues dans les élevages enquêtés, selon leur poids

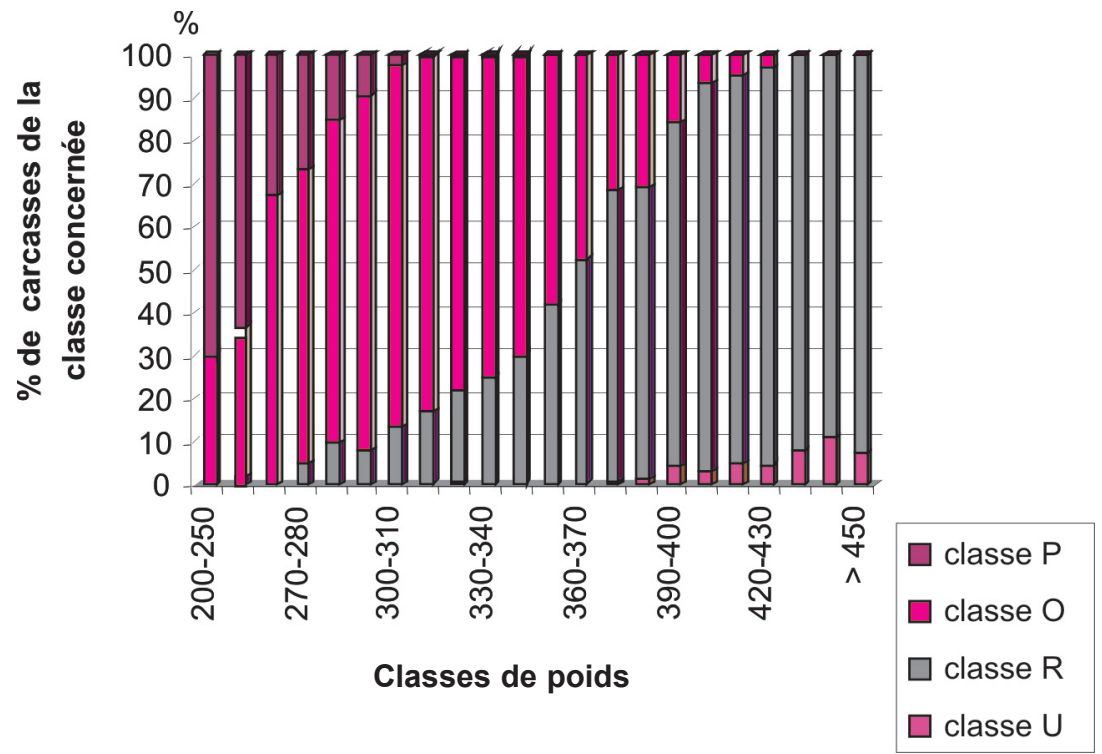

toriel depuis des modalités poids de carcasse faibles et conformation $\mathrm{O}$ ou $\mathrm{P}$ jusqu'aux modalités poids de carcasse élevés et conformation R (figure 4). Le troisième axe discrimine la saison d'abattage, opposant les modalités «été» et «hiver». La variable «âge» est peu structurante, sauf pour la modalité «vache de plus de 10 ans» (troisième axe). Quatre profils de performances ont été définis :

P1 «vaches légères et peu conformées» : 435 vaches formant deux sousgroupes à proximité des modalités de poids carcasse «320 - $340 \mathrm{~kg}$ » et «inférieur à $320 \mathrm{~kg}$ », ainsi que des modalités de classement $\mathrm{O}=$ et $« \mathrm{O}$ - à $\mathrm{P} »$.

P2 «vaches ordinaires» : 1830 vaches avec une position intermédiaire dans le plan, proches du centre du graphique et des modalités poids carcasse «320 - $340 \mathrm{~kg}$ ) ainsi que conformation $\mathrm{O}+$.

P3 «vaches lourdes et bien conformées» : 458 vaches proches des modalités poids carcasse «entre 370 et 400 $\mathrm{kg}$ » et conformation $\mathrm{R}$.

Figure 4. AFC : carte des modalités et profils de performances issus de la classification.

\begin{tabular}{|c|c|c|c|c|c|}
\hline \multirow{2}{*}{ Variables } & \multicolumn{5}{|c|}{ Modalités } \\
\cline { 2 - 6 } & $\mathbf{1}$ & $\mathbf{2}$ & $\mathbf{3}$ & $\mathbf{4}$ & $\mathbf{5}$ \\
\hline Age & $<4 a n s$ & $4-5$ ans & $6-9$ ans & $>=10$ ans & \\
\hline Poids (kg) & Inf 320 & $320-339$ & $340-369$ & $370-399$ & $>=400$ \\
\hline Classement & E - U - R & O plus & O égal & O moins - P & \\
\hline $\begin{array}{c}\text { Saison } \\
\text { d'abattage }\end{array}$ & $\begin{array}{c}\text { Décembre- } \\
\text { Février }\end{array}$ & Mars-Mai & Juin-Août & $\begin{array}{c}\text { Septembre- } \\
\text { Novembre }\end{array}$ & \\
\hline
\end{tabular}

P4 «vaches très lourdes et bien conformées» : 668 vaches proches des modalités poids carcasse «supérieur à $400 \mathrm{~kg}$ » et classement commercial R.

Ces quatre profils caractérisent des performances très différentes avec un écart entre extrêmes de $80 \mathrm{~kg}$ de poids de carcasse et de plus d'une classe de conformation. Nous nous proposons de tenter d'expliquer ces écarts par des différences de pratiques d'élevage.

\section{2 / Des pratiques d'élevage discriminées avant tout par la finition}

Les 25 exploitations enquêtées ont toutes une orientation polyculture-élevage laitier (tableau 1). En outre, 5 exploitations ont un troupeau allaitant de race Charolaise, support d'une production de vaches de réforme et de broutards repoussés ou de jeunes bovins. La production de viande issue du troupeau laitier revêt différentes formes : 
Tableau 1. Description de l'échantillon enquêté.

\begin{tabular}{|c|c|}
\hline Moyenne & Etendue \\
\hline SAU (ha) : 240 pour 2,57 UTH & 2 à moins de 100,3 à plus de 400 ha \\
\hline SFPISAU $(\%): 53$ & 4 à moins de 40,5 à plus de $60 \%$ \\
\hline SCOPISAU $(\%): 56$ & 5 à moins de 40,1 à plus de $60 \%$ \\
\hline Quota (litres) : 286000 & 4 à moins de 200000,4 à plus de $400000 \mathrm{~L}$ \\
\hline Effectif vaches laitières : 47 & \\
\hline $\begin{array}{l}\text { Productivité laitière : } 6200 \\
\text { (kg lait/vache/an) }\end{array}$ & 7 à moins de 40,7 à plus de 50 vaches \\
\hline TB et TP $(g / l): 40$ et 33,7 & 6 à moins de 6000,5 à plus de $7000 \mathrm{~kg}$ \\
\hline Poids carcasse vaches réforme : $358 \mathrm{~kg}$ & 4 à moins de $320 \mathrm{~kg}, 4$ à plus de $380 \mathrm{~kg}$ \\
\hline
\end{tabular}

autre production : 1 atelier vaches allaitantes dans 5 exploitations.

- Uniquement l'engraissement de vaches de réforme : 5 élevages

- Engraissement de vaches de réforme et de jeunes bovins : 10 élevages

- Engraissement de vaches de réforme et de bœufs : 6 élevages

- Engraissement de vaches de réforme, de jeunes bovins et de bœufs : 4 élevages

Le premier axe factoriel issu de l'AFCM oppose les élevages ne pratiquant ni tarissement ni finition au reste de l'échantillon (figure 5). Le second axe discrimine les élevages selon les pratiques de finition (finition de toutes les vaches, finition avec le troupeau laitier, finition variable selon les saisons), de rationnement du troupeau (composition de la ration de base) ainsi que du niveau laitier. Enfin, le troisième axe discrimine la variabilité des pratiques alimentaires de finition selon la saison.

Une classification ascendante hiérarchique réalisée sur les coordonnées de ces axes a permis de regrouper les élevages en 5 catégories (figure 5) :

\section{Type 1 : jamais de finition (4 éleva- ges)}

Dans ce groupe, $38 \%$ des vaches commercialisées ont un état d'engraissement de la carcasse noté 1 ou 2. La conduite d'élevage apparaît très variable selon les exploitations, tant en ce qui concerne les pratiques alimentaires (de 0 à $85 \%$ de maïs dans la matière sèche de la ration de base hivernale, de 1,1 à $1,8 \mathrm{~T}$ de concentrés/vache/an), que dans les perfor- mances laitières obtenues (de 5000 à $7000 \mathrm{~kg}$ de lait/vache/an). Le fait de ne jamais engraisser les vaches de réforme est volontaire et s'explique par la trop faible valorisation commerciale attendue (deux exploitations) ou par une spécialisation laitière à des fins de sélection (une exploitation), mobilisant toutes les ressources alimentaires disponibles. Dans le dernier cas, cette situation apparaît subie et liée notamment à un manque de places en bâtiments. tés de ces pratiques sont décrites dans la figure 2.
Type 2 : finition en faible proportion, ressources fourragères limitées (5 élevages)

Ces élevages se distinguent par une productivité laitière du troupeau inférieure à $6000 \mathrm{~kg} / \mathrm{vache} / \mathrm{an}$, par le choix des éleveurs de ne tarir et n'engraisser qu'une faible proportion des vaches de réforme ainsi que par des pratiques de finition adaptées à l'état corporel de la vache. La conduite alimentaire hivernale du troupeau laitier repose sur du foin seul (trois élevages) ou associé à de l'ensilage de maïs représentant $2 / 3$ des fourrages totaux (deux élevages).

L'alimentation estivale est constituée de pâturage, à raison de 40 à 60 ares/vache selon les élevages, complété par du maïs ensilage (deux exploitations) ou de l'herbe ensilée. Les éleveurs de ce groupe estiment leurs ressources fourragères insuffisantes eu égard aux besoins des animaux. Face aux ruptures de stock fourrager, ils choisissent de rationner les jeunes (génisses et bœufs de 18 mois) et de ne pas finir environ la moitié de leurs vaches réformées. Le quart des vaches vendues a un état d'engraissement insuffisant $(<=2)$. Les pratiques alimentaires en finition varient selon la saison et les ressources disponibles : pâture non complémentée en été, foin/paille ou parfois ensilage de maïs en hiver (un cas) avec une complémen-

Figure 5. Représentation de la typologie des élevages sur le plan factoriel représenté par les deux premiers axes de l'AFCM réalisée sur 9 pratiques d'élevage. Les modali-

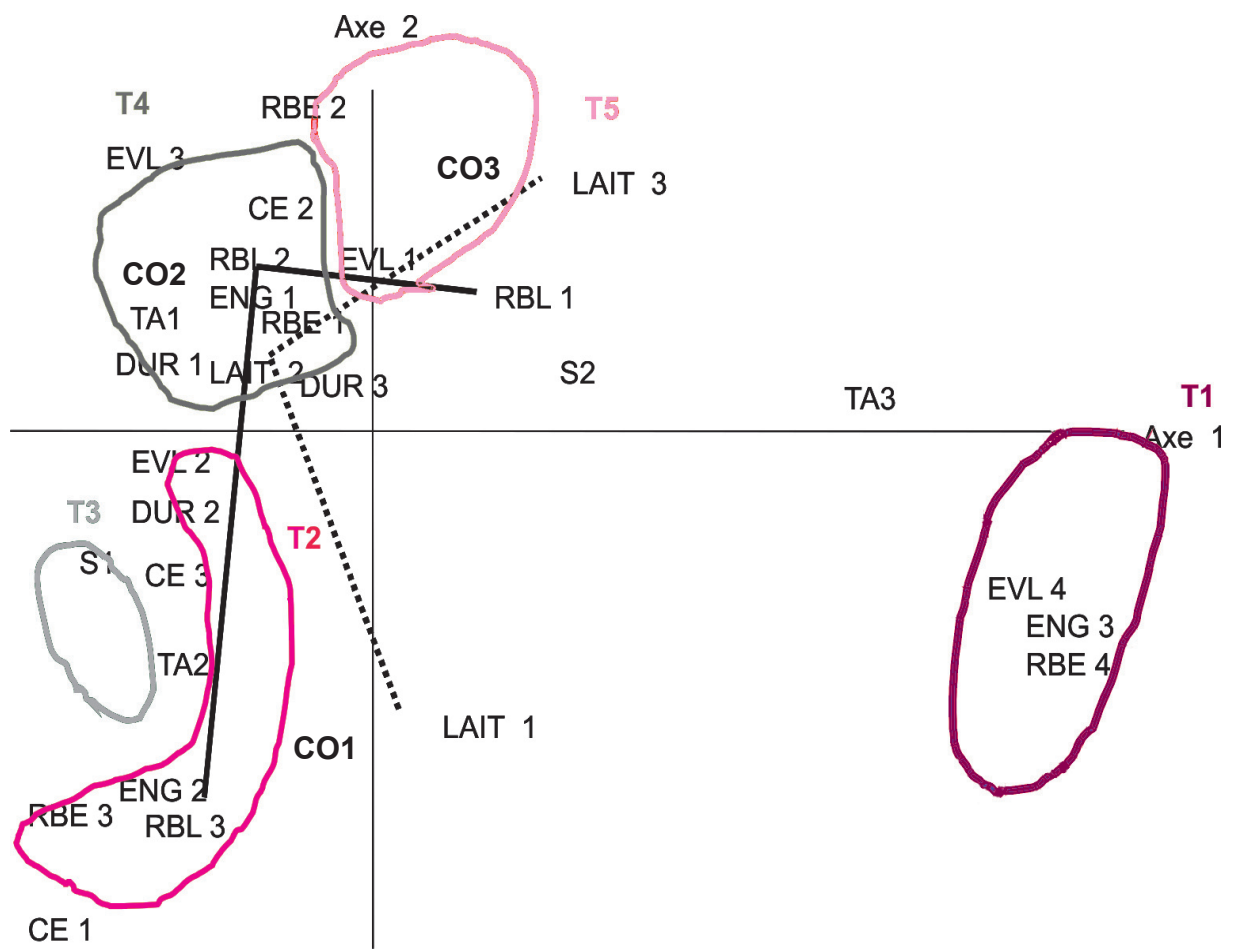


tation variant selon le fourrage distribué de 2 à $7 \mathrm{~kg} / \mathrm{j}$ de céréales et de 1,5 à $2 \mathrm{~kg} / \mathrm{j}$ de tourteau durant 2 à 3 mois.

Type 3 : tarissement et finition systématiques à base de ressources herbagères ( 3 élevages)

Ces élevages sont très homogènes en termes de niveau laitier moyen $(6000$ $\mathrm{kg} / \mathrm{vache} / \mathrm{an}$ ) et de pratiques de finition des vaches de réforme : tarissement systématique, séparation du troupeau laitier, conduite alimentaire variable selon la saison, mais toujours à partir de ressources herbagères. La conduite alimentaire du troupeau laitier repose exclusivement sur l'herbe sous forme de pâturage en été et conservée en foin en hiver. La consommation annuelle de concentrés est inférieure à 1 T/vache/an, à l'exception d'un élevage qui dépasse 1,5 $\mathrm{T}$.

Après tarissement, la finition des vaches se déroule soit en été à l'herbe, avec un complément de 2 à $3 \mathrm{~kg} / \mathrm{j}$ de céréales durant 3 mois, soit en automne-hiver, avec une ration de foin complétée par 3 à $5 \mathrm{~kg} / \mathrm{j}$ de céréales pendant au moins 3 mois. L'état corporel initial estimé visuellement par l'éleveur est le paramètre qui détermine la durée de finition (de 2 à 6 mois selon les vaches) car aux dires des éleveurs «des vaches maigres ont besoin de temps pour se refaire». Les pratiques systématiques de tarissement et finition observées dans ce groupe sont à mettre en lien avec la conduite alimentaire à dominante herbagère, à l'origine d'un état corporel souvent assez faible.

Type 4: finition systématique après tarissement à base d'ensilage de maïs (8 élevages)

La productivité laitière de ces élevages varie de 5500 à $6500 \mathrm{~kg} / \mathrm{vach}$, avec une ration de base hivernale composée d'ensilage de maïs (jusqu'à 2/3 de la matière sèche), d'autres fourrages (ensilage d'herbe ou foin) et une consommation plutôt modérée de concentrés (1060 kg/vache/an).

Les vaches réformées sont systématiquement taries, puis séparées du troupeau laitier. Deux types de finition peuvent alors être distingués : (i) une conduite alimentaire invariable tout au long de l'année (quatre élevages) : finition soit à l'attache (deux élevages), soit en stabulation libre avec une ration à base de maïs ensilage, complété de foin, parfois d'ensilage d'herbe (deux élevages), de 2 à $4 \mathrm{~kg} / \mathrm{j}$ de céréales et de 1 à $2 \mathrm{~kg}$ de tourteau durant une période qui varie entre 1 et 4 mois selon l'état corporel initial et la morphologie de la vache (ii) une conduite alimentaire à l'auge en automne-hiver et à l'herbe en printemps-été (quatre élevages), avec $3 \mathrm{~kg}$ de céréales/j pendant un à deux mois. La durée de finition plus courte qu'en hiver est à mettre en relation avec le stade de lactation des vaches réformées au printemps, généralement plus avancé. Le tarissement systématique est ici associé à un meilleur rendement boucher.

Type 5 : finition systématique en général sans tarissement et conduite alimentaire intensive (5 élevages)

Ce groupe se caractérise par une conduite alimentaire de type «maïsconcentrés» et des pratiques de finition constantes selon les vaches réformées et les saisons. Ainsi, la mise en œuvre d'une ration complète (deux exploitations) ou semi-complète (trois exploitations) tout au long de l'année, à base de maïs ensilage (pour plus de $70 \%$ de la matière sèche de la ration) et une consommation de concentrés de 1676 $\mathrm{kg} / \mathrm{vache} / \mathrm{an}$, sont associées à une production laitière de 7300 kg/vache/an.

La finition des vaches réformées est réalisée en les maintenant au sein du troupeau laitier avec une ration couvrant 25 à $28 \mathrm{~kg}$ de lait/jour, très supérieure à leurs besoins, sachant qu'elles sont plutôt en fin de lactation. Les éleveurs de ce groupe estiment en effet que le tarissement avant réforme ne se justifie plus économiquement, avec un gain estimé à $10 \mathrm{~kg}$ de carcasse supplémentaires en regard d'une période improductive de deux mois. Le tarissement des vaches de réforme n'est alors pratiqué que pour des vaches âgées ou très maigres. Le taux de réforme de ces troupeaux est très élevé (39\%) et l'âge moyen des vaches réformées dans ce type d'élevage est le plus faible de l'échantillon (tableau 2), la différence étant significative par rapport aux types 2 et 4 .

\section{3 / Discussion : la mixité comme lien entre pratiques et performances}

\section{1 / Caractéristiques de la population étudiée}

Le poids de carcasse des vaches Simmental Française est un peu supérieur à celui des vaches Montbéliarde $(+24 \mathrm{~kg})$ et Holstein $(+20 \mathrm{~kg})$ selon des données recueillies dans les mêmes conditions (données d'abattage,
Bastien 2001) mais inférieur à celui de la race Normande $(-12 \mathrm{~kg})$, avec toutefois un âge plus avancé $(+0,7$ ans que les vaches Normande). Ces données sont assez conformes aux appréciations d'experts de la filière (négociants et bouchers) qui situent la race Simmental Française actuelle à un niveau intermédiaire entre la Montbéliarde et la Charolaise (Gaillard et Fauriat 2003). Cette position intermédiaire est également observée dans le cas des mâles engraissés de type Simmental dont les performances apparaissent inférieures à celles de races à viande (Dufey et al 2002) mais supérieures à celles de taurillons laitiers (Szücs et al 2001). Les résultats de croissance des veaux avant sevrage situent la Simmental au-dessus des races anglaises et des races allaitantes spécialisées, en raison de son potentiel laitier (Jenkins et Ferrell 1992). Une expérimentation portant sur la finition de vaches de réforme a mis en évidence chez les vaches Simmental certains caractères laitiers tels qu'une capacité d'ingestion importante et un rendement à l'abattage peu élevé mais a également montré leur bonne capacité de reprise de poids au cours de l'engraissement, surtout sous forme de muscle, cette caractéristique les rapprochant des vaches de races à viande spécialisées (Dumont et al 1994).

Toutefois, les performances moyennes à l'abattage masquent une grande variabilité qui s'explique par des modes de production différents. La production de viande par les vaches de réforme ne revêt pas la même importance selon les types d'élevage identifiés. Nous pouvons ainsi souligner la gamme des pratiques d'élevage observées, allant du non-engraissement des vaches jusqu'à la mobilisation de ressources alimentaires telles que l'ensilage de maïs et la complémentation en concentrés favorisant une expression plus complète du potentiel boucher. La première situation, qui se traduit par une valorisation aléatoire du potentiel boucher, avec des performances «en l'état» des vaches réformées en cours de lactation, relève soit d'un choix délibéré de l'éleveur privilégiant une sélection laitière soit plus fréquemment de l'absence de ressources en fourrages ou en bâtiment.

La question du tarissement paraît diviser les éleveurs quant à son influence sur le poids de carcasse. Ceux qui le pratiquent y voient un facteur favorable à la finition et la garantie d'un poids de carcasse élevé («une vache non tarie, même si elle est finie, ne pèse pas»). Les autres jugent cette période «improductive, non 
Tableau 2. Performances des vaches de réforme et pratiques de choix des reproducteurs selon les types d'élevages identifiés (T1 à T5).

\begin{tabular}{|c|c|c|c|c|c|}
\hline \multirow[b]{2}{*}{$\begin{array}{l}\text { Performances } \\
\text { d'abattage }\end{array}$} & \multicolumn{5}{|c|}{ Types d'élevage } \\
\hline & $\begin{array}{c}\text { Type } 1 \\
\text { Absence } \\
\text { de finition }\end{array}$ & $\begin{array}{c}\text { Type } 2 \\
\text { Finition d'une } \\
\text { partie des } \\
\text { animaux }\end{array}$ & $\begin{array}{c}\text { Type } 3 \\
\text { Finition } \\
\text { ressources } \\
\text { herbagères }\end{array}$ & $\begin{array}{c}\text { Type } 4 \\
\text { Finition } \\
\text { tarissement } \\
\text { maïs }\end{array}$ & $\begin{array}{c}\text { Type } 5 \\
\begin{array}{c}\text { Finition non tarissement } \\
\text { maïs }\end{array}\end{array}$ \\
\hline Age moyen (ans) & $6,2+/-2,6$ & $6,6+/-2,6$ & $6+1-2$ & $6,5+/-1,1$ & $5,8+/-2,1$ \\
\hline $\begin{array}{l}\text { Poids moyen de } \\
\text { carcasse }(\mathrm{kg})\end{array}$ & $312+/-35$ & $334+/-32,5$ & $356+/-36$ & $365+/-38$ & $382+/-44$ \\
\hline $\begin{array}{l}\text { Conformation } \\
\text { moyenne des } \\
\text { carcasses }\end{array}$ & proche de $\mathrm{O}=$ & proche de O+ & $\begin{array}{l}\text { entre } \mathrm{O+} \\
\text { et } \mathrm{R}-\end{array}$ & $\begin{array}{l}\text { entre O+ } \\
\text { et R- }\end{array}$ & $\begin{array}{l}\text { entre } R \\
\text { et } R=\end{array}$ \\
\hline $\begin{array}{l}\% \text { carcasses en état } \\
\text { de gras - note } 1 \text { et } 2\end{array}$ & 38 & 24 & 2,8 & 2,5 & 0,8 \\
\hline $\begin{array}{l}\text { Profil de } \\
\text { performances } \\
\text { bouchères }\end{array}$ & P1 & $\mathrm{P} 1$ et $\mathrm{P} 2$ & P2 et P3 & P2, P3 et P4 & P3 et P4 \\
\hline $\begin{array}{l}\text { Taux moyen de } \\
\text { réforme (\% VL) }\end{array}$ & 24,5 & 28,8 & 31,6 & 27,8 & 38,4 \\
\hline \multicolumn{6}{|c|}{ Pratiques de sélection } \\
\hline $\begin{array}{l}\text { Prise en compte de } \\
\text { critères laitiers } \\
\text { Seuil de tri } \\
\text { primipares, aux } 1^{\text {ers }} \\
\text { contrôles laitiers }\end{array}$ & $\begin{array}{c}+++ \\
15 \mathrm{~kg} \\
\text { (2 élevages) } \\
20 \mathrm{~kg} \\
\text { (2 élevages) } \\
\end{array}$ & $\begin{array}{c}+++ \\
15 \mathrm{~kg}\end{array}$ & $\begin{array}{c}++ \\
\text { de } 12 \\
\text { à } 20 \mathrm{~kg}\end{array}$ & $\begin{array}{c}++ \\
\text { de } 15 \\
\text { à } 20 \mathrm{~kg}\end{array}$ & $\begin{array}{c}+++ \\
\text { de } 15 \\
\text { à } 23 \mathrm{~kg}\end{array}$ \\
\hline $\begin{array}{l}\text { Prise en compte } \\
\text { des critères } \\
\text { fonctionnels }\end{array}$ & $\stackrel{+}{+}+\stackrel{ }{\text { taux cellulaire }}$ & $\begin{array}{c}++ \\
\text { taux cellulaire } \\
\text { fertilité }\end{array}$ & $\begin{array}{l}+ \\
\text { Régularité } \\
\text { de } \\
\text { production } \\
\text { (fertilité) }\end{array}$ & $\begin{array}{l} \\
+ \\
\text { fécondité } \\
\text { régulière } \\
\text { résistance } \\
\text { maladies } \\
\end{array}$ & $\begin{array}{c}++ \\
\text { facilité de traite } \\
\text { conformation } \\
\text { mamelle } \\
\text { taux cellulaire } \\
\end{array}$ \\
\hline $\begin{array}{l}\text { Prise en compte de } \\
\text { la morphologie } \\
\text { bouchère }^{1}\end{array}$ & - & - & + & ++ & + \\
\hline
\end{tabular}

1 Postes musculature et développement.

justifiée par la valorisation commerciale actuelle de la viande». Cette dernière position est cohérente avec l'analyse de Meffe et al (2005) qui estiment que l'absence de tarissement avant réforme ne nuit pas forcement aux performances à l'abattage excepté dans le cas de vaches réformées en pleine lactation et abattues trop maigres.

En ce qui concerne la conduite alimentaire, l'augmentation de la part d'ensilage de maïs dans la ration tend à uniformiser les pratiques de finition entre les différentes saisons et entre les individus. En cas de ration complète ou semi-complète avec distribution de maïs toute l'année (élevages T5), les vaches réformées sont finies avec la ration du troupeau laitier et demeurent d'ailleurs dans ce troupeau en lactation jusqu'à leur vente. A l'opposé, dans les élevages à dominante herbagère (T3 et en partie T4), l'éleveur adapte ses pratiques en fonction des ressources disponibles, de la saison (pâturage $v s$ foin et concentrés) et de l'individu (ajustement de la ration et de la durée de finition en fonction de l'état corporel et de l'âge de la vache), ceci après un tarissement systématique. Cette pratique d'allongement de la durée de finition qui peut aller jusqu'à six mois, apparaît surprenante au regard des recherches conduites sur l'engraissement de vaches de race Normande (Béranger et al 1970) ou Charolaise (Dumont et al 1991) indiquant qu'au delà du 50ème jour, la reprise de poids est fortement ralentie et le surplus de carcasse obtenu est essentiellement composé de gras. Toutefois, dans le cas des types de finition T3 ou T4, cette pratique ne concerne que certains animaux et avec des rations bien moins énergétiques que dans les conditions expérimentales des auteurs cités, s'agissant de pâturage, d'ensilage d'herbe ou de foin. L'objectif est de reconstituer des vaches ayant un état corporel assez faible au début de l'engraissement, et de permettre un dépôt musculaire progressif favorisé par le déplacement au pâturage (Micol et al 1997).

Ces pratiques d'engraissement, différenciées en fonction des ressources disponibles exigent du temps, du suivi et un savoir-faire de l'éleveur. Il serait utile de les détailler davantage par une approche effectuée au niveau de l'animal dans le cadre d'un suivi avec plusieurs passages à des dates clés pour accéder aux variations saisonnières des pratiques (engraissement au pré, à l'étable...).

\section{2 / Différents degrés dans la valorisation de la mixité}

La mise en relation des performances bouchères avec les pratiques d'élevage et de finition qui leur sont associées révèle une grande diversité dans la valorisation du caractère mixte de la race (tableau 3). Il existe ainsi une différence de $70 \mathrm{~kg}$ de carcasse et d'environ une classe de conformation entre les éleveurs T1 vendant leurs vaches «en l'état» (312 kg, classement proche de O-) et les éleveurs T5 produisant les animaux les plus lourds (382 kg) et les mieux conformés $(\mathrm{R}-\mathrm{à} \mathrm{R}=)$. Les types de pratiques $\mathrm{T} 1$ et $\mathrm{T} 2$ sont associés respectivement à 38 et $24 \%$ de carcasses maigres contre moins de $3 \%$ pour les types de pratiques T3, T4 et T5 (tableau 2). L'existence de systèmes extrêmes du non-engraissement au tout-engraissement des vaches de réfor- 
Tableau 3. Répartition (en nombre d'exploitations) des types d'élevage identifiés à partir des pratiques de conduite, dans les différents profils de performances bouchères pour l'ensemble des exploitations enquêtées.

\begin{tabular}{|l|c|c|c|c|c|}
\hline & \multicolumn{5}{|c|}{ Cinq types de pratiques de finition (figure 5) } \\
\cline { 2 - 6 } & $\begin{array}{c}\text { T1 } \\
\text { Qubsence } \\
\text { Quatre Profils } \\
\text { de performances } \\
\text { bouchères (figure 4) }\end{array}$ & $\begin{array}{c}\text { T2 } \\
\text { Parfois } \\
\text { finition } \\
\text { ressources } \\
\text { limitées }\end{array}$ & $\begin{array}{c}\text { T3 } \\
\text { Toujours } \\
\text { finition } \\
\text { tarissement } \\
\text { ressources } \\
\text { herbagères }\end{array}$ & $\begin{array}{c}\text { T4 } \\
\text { Toujours } \\
\text { finition } \\
\text { tarissement } \\
\text { Maïs } \\
\text { dominant }\end{array}$ & $\begin{array}{c}\text { T5 } \\
\text { Toujours } \\
\text { finition } \\
\text { tarissement } \\
\text { rare } \\
\text { Tout maìs }\end{array}$ \\
\hline $\begin{array}{l}\text { P1 «vaches légères } \\
\text { et peu conformées» }\end{array}$ & 4 & 1 & & 3 & 1 \\
\hline P2 «vaches moyennes» & & 4 & 2 & 3 & 4 \\
\hline $\begin{array}{l}\text { P3 «vaches lourdes } \\
\text { et bien conformées» }\end{array}$ & & & 1 & 2 & \\
\hline $\begin{array}{l}\text { P4 «vaches très lourdes } \\
\text { et bien conformées» }\end{array}$ & & & & 3 & \\
\hline
\end{tabular}

me situe précisément la gamme de la mixité mise en œuvre par les éleveurs, révélant différentes logiques de fonctionnement des systèmes d'élevage mais peut-être également différentes perceptions et attentes des éleveurs. La corrélation positive observée entre les performances laitières et bouchères (figure 6) joue en faveur des éleveurs de type T5, disposant de ressources abondantes, avec une part dominante de maïs ensilage en vue d'obtenir des performances laitières élevées, selon des modes de conduite assez semblables à ceux observés chez des éleveurs de races laitières spécialisées. De fait, ces ressources, alliées à des conditions de logement, de suivi sanitaire souvent optimales, bénéficient également aux performances bouchères, dans la mesure où les vaches de réforme, non séparées du troupeau laitier reçoivent une ration supérieure à leurs besoins de production laitière, assurant leur finition. Le tri important des femelles, la sélection exercée sur des critères laitiers se traduisent par un taux de réforme supérieur à celui des autres types d'élevage (près de $40 \%$ ) et une part importante de vaches réformées jeunes $(60 \%$ à moins de 6 ans), source d'une production de viande généralement bien valorisée. La production de viande par la réforme apparaît comme le coproduit de la production laitière, dans un équilibre «haut», maximisant les deux aptitudes. On trouve dans ce groupe T5 des éleveurs sélectionneurs, attachés à la promotion de leur race, à l'expression du potentiel et à la progression des per-
Figures 6. Liaison entre performances laitières et bouchères des troupeaux enquêtés.

\section{Liaison entre production laitière et poids de carcasse}

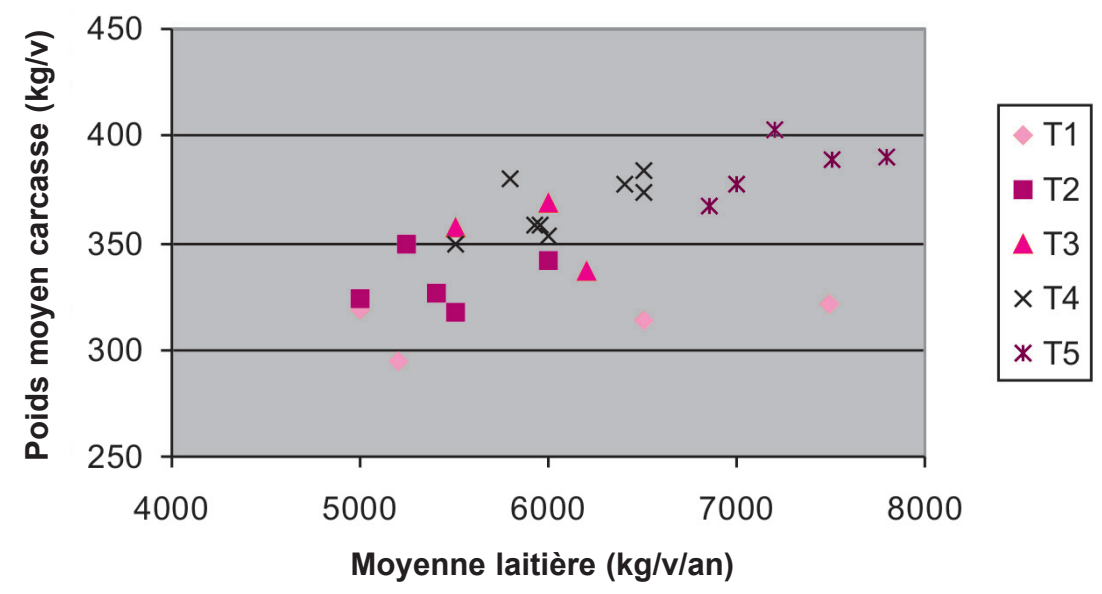

Liaison entre production laitière et conformation de la carcasse

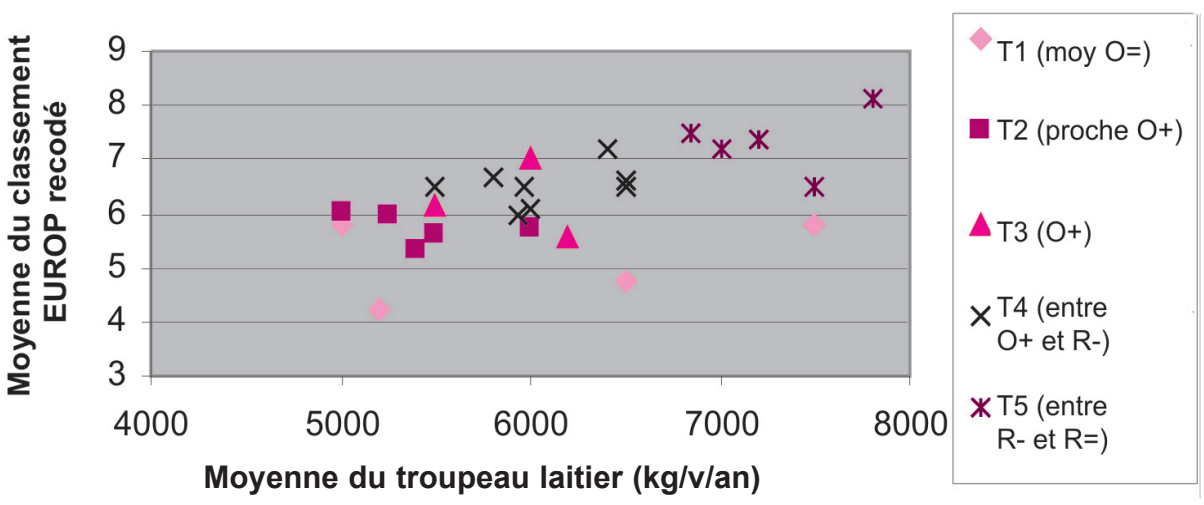


formances, malgré des coûts probablement élevés, notamment en ce qui concerne l'alimentation. Le fait qu'il s'agisse d'éleveurs sélectionneurs, fiers de leur race n'est sans doute pas anodin dans leur capacité d'intensification, source d'une progression continue des performances.

\section{Les éleveurs plus «herbagers»} (types T3 et T4), semblent avoir trouvé une manière de s'adapter aux contraintes de leur système, limitant le niveau des performances tant en lait qu'en viande. L'équilibre lait-viande auquel ils aboutissent s'exprime par un niveau de performances plus modeste que celui des élevages précédents (des poids moyens de carcasse respectivement de 356 et $365 \mathrm{~kg}$, non significativement différents, une conformation moyenne entre $\mathrm{O}+$ et $\mathrm{R}$, une productivité laitière de 6000 $\mathrm{kg} /$ vache). Ces élevages disposent de ressources fourragères qui ne sont pas toujours bien adaptées à une finition poussée des animaux, comme d'ailleurs à une productivité laitière élevée. La mixité de la race est ici justifiée en cohérence avec le système d'élevage, à travers un degré d'équilibre «moyen» comparé à l'équilibre «haut» des élevages T5. Les performances bouchères viennent certes en complément de la production laitière, mais sous une forme différente que celle des élevages T5 : elles sont obtenues grâce à une combinaison de pratiques dont font partie l'alimentation, mais aussi le tarissement, l'allongement possible de la durée de finition en fonction de l'animal et de son état corporel initial, la sélection sur la morphologie, dans le cadre d'ajustements parfois complexes, résultant de choix individuels.

Il est intéressant de noter que la forme de mixité ainsi mise en œuvre, au sein d'exploitations herbagères notamment, avec une race adaptée au système, apparaît finalement assez proche de la logique de fonctionnement, majoritairement recherchée par les nouveaux éleveurs Simmental de la zone d'extension du berceau racial, à la recherche d'un cheptel susceptible de s'adapter à leurs contraintes (exploitations situées en conditions pédoclimatiques limitantes pour la culture de maïs-fourrage, structure exiguë, droits à produire peu élevés...). C'est le cas du développement de la race dans le département de l'Aveyron, ou plus récemment dans certaines zones herbagères de l'Est (Meuse) ou de l'Ouest (Ille-et- Vilaine).

Figure 7. Liaison entre effet troupeau et poids moyen de carcasse des vaches de réforme.

\section{Liaison entre effet troupeau et poids de carcasse}

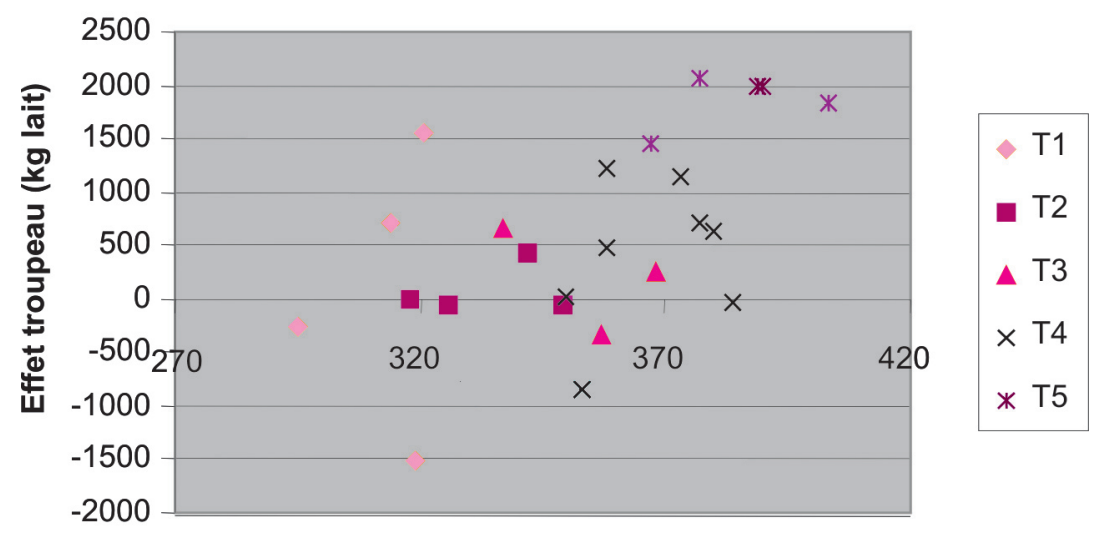

Poids de carcasse $(\mathrm{kg})$

La prise en compte de l'effet troupeau renforce la distinction entre les types T3 et T4 d'une part et le type T5 d'autre part (figure 7). Un poids moyen de carcasse de 350 à $400 \mathrm{~kg}$ est associé à un effet troupeau variant de - 500 à $+1500 \mathrm{~kg}$ de lait. Il apparaît ainsi dans les groupes T3 et T4 que des élevages de productivité laitière modeste $(6000$ $\mathrm{kg}$ de lait /vache/an) avec un faible effet troupeau (inférieur à $500 \mathrm{~kg}$ de lait), expriment un bon niveau de performances bouchères (poids carcasse d'au moins $370 \mathrm{~kg}$ ), permis par une stratégie de sélection intégrant des critères de morphologie bouchère.

Les élevages T1 et T2 présentent des traits communs de fonctionnement malgré des performances bouchères supérieures pour les seconds, résultant d'une finition de certains animaux $(+22 \mathrm{~kg}$ de carcasse, $+1 / 4$ de classe de conformation). En effet, pour ces deux groupes, la production de viande ne semble pas avoir d'importance à la fois dans l'affectation des ressources mais aussi dans les pratiques de sélection. L'évolution des systèmes T2 pose question : les déficits de ressources observés sont-ils conjoncturels et susceptibles d'ajustements, avec la perspective d'évoluer vers une logique T3 ou T4 ? ou au contraire plus durables, ce qui avec la priorité portée sur le troupeau laitier les rapprocherait des systèmes T1?

Les différentes combinaisons de pratiques d'élevage et de niveau de performances bouchères configurent de notre point de vue un gradient d'expression de la mixité, avec la possibilité d'y déplacer un curseur situant le niveau d'équilibre exprimé par l'éleveur en fonction de ses objectifs et de ses contraintes. Cette capacité de déplacement (réglage) de ce curseur peut être alors considérée comme un atout des races mixtes par rapport aux races spécialisées. Ce «modèle de la mixité» mériterait d'être testé et validé avec d'autres races et dans d'autres situations.

\section{3 / Conséquences pour la sélec- tion}

Tous les éleveurs déclarent retenir en priorité les critères laitiers dans leurs objectifs de sélection. En revanche, il existe une certaine diversité dans la prise en compte de critères fonctionnels ou de morphologie bouchère (tableau 2 ). Les éleveurs des types T1 et T2 sont attentifs aux critères de quantité de lait et de qualité associés (résistance aux mammites, fertilité), mais ne prennent pas en compte la morphologie ni la conformation bouchère.

Les éleveurs de type T3 recherchent un animal équilibré «produisant 6000 $\mathrm{kg} /$ lactation, robuste, facile à conduire avec une fécondité régulière (un veau/an)». Ceux de type T4 accordent également de l'importance à un ensemble de critères laitiers et fonctionnels, insistant toutefois sur la morphologie «une vache sans problème, qui se reproduit facilement et de bonne morphologie». Ces éleveurs sont ceux qui déclarent accorder le plus d'intérêt à la morphologie bouchère. Enfin, les éleveurs du type T5 sont également attentifs aux critères fonctionnels et de 
morphologie bouchère, mais affirment une nette priorité laitière en situant le seuil de production laitière à un niveau supérieur à celui des types T3 et T4 (seuil de tri des primipares aux premiers contrôles laitiers à $20 \mathrm{~kg}$ de lait en moyenne et objectif de productivité moyenne annuelle à $7000 \mathrm{~kg}$ de lait/vache/an). Il existe une corrélation positive $\left(\mathrm{r}^{2}=0,58\right)$ entre la productivité laitière annuelle et le poids moyen de carcasse des vaches réformées, à l'échelle du troupeau. De même, les troupeaux ayant les vaches réformées les mieux conformées, sont aussi ceux qui produisent le plus de lait $\left(\mathrm{r}^{2}=0,50\right.$; $\mathrm{P}=0,01$ entre la productivité laitière et la conformation moyenne de la carcasse). Le croisement entre les performances bouchères et l'effet troupeau calculé par l'Institut de l'Elevage va dans le même sens : le poids de carcasse augmente avec l'effet troupeau, avec cependant une certaine dispersion des points notamment pour les types d'élevages $\mathrm{T} 1$ et $\mathrm{T} 4$ (figure 7 ).

La connaissance des différents modes de conduite des vaches de race Simmental française met bien en évidence la difficulté inhérente à la mise en œuvre d'un programme de sélection homogène, orienté vers la mixité laitviande. De fait, les modes de production décrits traduisent des attentes différentes des éleveurs dans la prise en compte des aptitudes bouchères. Leurs attentes sur la conformation semblent être plus fortes dans les systèmes herbagers (T3), là où les conditions de finition sont plus fréquemment limitantes. En revanche, les éleveurs T5 privilégient le format, le potentiel de croissance, plus compatibles avec la priorité génétique accordée à la progression des caractères laitiers, que les critères de conformation musculaire. L'équilibre recherché entre les deux composantes lait et viande ne signifie pas la maximisation simultanée du potentiel de chacune mais bien un compromis entre d'une part, un progrès laitier car la vache est d'abord et toujours traite, d'autre part, des critères de croissance et une conformation minimale, base de valorisation sur un marché, non spécifique, de femelles laitières.

\section{Conclusion}

Cette étude a permis de situer les performances bouchères à l'abattage de vaches de réforme Simmental Française, «utilisée» comme exemple de race mixte et de dégager quatre profils de performances selon le poids et la conformation des carcasses. Des enquêtes en exploitation ont permis de distinguer cinq types de pratiques de conduite du troupeau laitier et de finition des vaches de réforme. Le croisement entre les deux approches met en évidence les relations qui existent entre elles, avec notamment une corrélation positive entre la pratique du tarissement, la nature des ressources alimentaires mobilisées, le niveau de production laitière et l'expression du potentiel boucher. La relation entre performances laitières et bouchères indique que les éleveurs ayant les plus fortes productivités laitières sont aussi ceux qui produisent les carcasses les plus lourdes et les mieux conformées, maximisant l'expression des deux aptitudes. Toutefois, il s'avère aussi que de bonnes performances bouchères peuvent être associées à une productivité laitière plus modeste. Ces deux voies révè- lent des attentes différentes des éleveurs concernant le type d'animal.

La relation ainsi mise en évidence entre pratiques et performances (la mobilisation de données d'abattage étant une des originalités de l'étude), peut-être considérée comme la possibilité pour les éleveurs de déplacer un curseur le long d'un gradient d'expression des performances zootechniques, sans que les extrémités de ce gradient puissent être jugées en termes d'efficacité supérieure ou moindre. La validation de cette hypothèse nécessiterait un élargissement à d'autres races dans d'autres contextes, ainsi qu'une évaluation économique des différentes combinaisons identifiées (i.e. différentes positions du curseur). Il s'agirait de tester un modèle de la mixité des races, alternatif à la spécialisation, dont l'intérêt serait de permettre une plus grande adaptabilité aux objectifs et aux contraintes des éleveurs.

\section{Remerciements}

Cette étude s'est déroulée dans le cadre du projet de recherche inter-unités INRA-OPAL, portant sur les modes d'organisation des populations animales locales en lien avec des projets d'acteurs. Nous tenons à remercier les membres du projet OPAL et plus spécialement F. Casabianca et A. Audiot pour leur aide méthodologique et leur questionnement, ainsi que les éleveurs qui ont accepté d'évoquer leurs pratiques d'élevage et de transmettre leurs données. En outre, cette étude a bénéficié du soutien financier du Conseil Scientifique 5b de l'ENESAD.

\section{Références}

Audiot A., Bouche R., Brives H., Casabianca F., Gaillard C., Roche B., Trift N., Steyaert P., 2004. Populations animales locales et produits de qualité : comment valoriser transforme la ressource génétique ? 5ème Coll. Nat.BRG, Lyon, novembre 2004, 577-592.

Bastien D., 2001. Incidence de l'âge d'abattage des vaches sur la qualité des carcasses et des viandes. C.R. final, Institut de l'Elevage, 34 p + annexes.

Béranger C., Negrin M., Malterre C., 1970. Evolution du gain de poids vif et de l'état d'engraissement des vaches taries au pâturage. Ann. Zootech., 19 (1), 53-66.

Cristofini B., Deffontaines J.P., Raichon C., 1978. Pratiques d'élevage en Castagniccia. Exploitation d'un milieu naturel et rural en Corse. Etudes Rurales, 71-72, 89-109.
Dufey P.A., Chambaz A., Morel I., Chassot A., 2002. Performances d'engraissement de boeufs de six races à viande, Rev. Suisse Agric., 34 (3), 117-124.

Dumont R., Roux M., Agabriel J., Touraille C., Bonnemaire J., Malterre C., Robelin J., 1991. Engraissement des vaches de réforme de race Charolaise, INRA Prod. Anim., 4 (4), 271-286.

Dumont R., Masuez A., Roux M., Gaillard C., 1994. Aptitude à la production de viande de vaches de réforme de race Simmental Française, caractérisation des qualités des carcasses et des viandes. C.R. première année d'expérimentation 1992-1993, INRA et ENESAD, 13p.

France Contrôle Laitier, 2004. Les chiffresclés du Contrôle Laitier bovin, 8p.
Gaillard C., 1995. Histoire et actualité d'une race bovine mixte : la race Simmental française dans les systèmes d'élevage de Haute-Marne. Thèse de Doctorat INA PG- ENESAD, 177p + annexes.

Gaillard C., Fauriat C., 2003. Valorisation bouchère des animaux de race Simmental Française, Renc. Rech. Rumim., 10, 256.

Institut de l'Elevage, INRA, 2004. Note IBL2004-4, calcul de l'IBOVAL.

Jenkins T.G., Ferrell C.L., 1992. Lactation characteristics of nine breeds of cattle fed various quantities of dietary energy. J. Anim. Sci., 70, 1652-1660.

Landais E., 1987. Recherches sur les systèmes d'élevage, questions et perspectives. Document 
de travail, URSAD Versailles- Dijon- Mirecourt, Versailles, INRA, 75p.

Meffe N., Bastien D., Chatelin Y.M., 2005. Abattage des vaches laitières en lactation: quantification du phénomène et conséquences pour les abattoirs. C.R. final, 170532019, Institut de l'Elevage, 103p.
Micol D., Dedieu B., Agabriel J., Béranger C., 1997. Adaptation de la production de viande bovine aux systèmes extensifs d'élevage. Fourrages, 149, 3-20.

Règlement CEE, 1981. Journal Officiel L 293 du 13/10/1981, 6
Szücs E., Ender B., Papstein H.J., Nürnberg G., Ender K., 2001. Vergleich des Schlacht und Nährwertes sowie der Fleischbeschaffenheit von Jungbullen der Rassen Deutsches Fleckvieh und Deutsches Holstein (Schwarzbunte) im Verlauf des Wachstums.1.Mitteilung: Wachstum und Schlachtkörperzusammensetzung Züchtungskunde, 73 (1), 33-44.

\section{Résumé}

L'objectif de ce travail a été d'étudier les performances bouchères de vaches de réforme de race Simmental Française à partir de données d'abattage, puis de les mettre en relation avec les modes de production des animaux appréhendés en amont à travers des enquêtes en élevage.

Une Analyse Factorielle Multiple (AFCM) réalisée sur les caractéristiques d'abattage de $\mathbf{2 7 4 3}$ vaches de réforme issues de 122 élevages de trois départements (Côte d'Or, Haute-Marne, Jura) a permis de définir quatre profils de performances bouchères, en fonction du poids de carcasse et de la conformation.

Une enquête directe dans vingt-cinq élevages, choisis par stratification, a conduit à distinguer par AFCM cinq profils d'exploitations en fonction des pratiques mises en ouvre et des ressources alimentaires mobilisées particulièrement lors de la finition.

Le croisement entre ces deux typologies met en évidence une grande diversité dans la valorisation du caractère mixte de la race. La gamme des pratiques d'élevage observées, va du non-engraissement jusqu'au tout-engraissement des vaches de réforme avec des écarts de performances entre groupes extrêmes de $70 \mathrm{~kg}$ de poids de carcasse et de plus d'une classe de conformation. Les performances les plus élevées (carcasse de poids $>=380 \mathrm{~kg}$, classée $R$ - à $R=$ ) sont associées à une forte mobilisation de ressources alimentaires, notamment l'ensilage de maïs mais également à la mise en ouvre de pratiques spécifiques telles que le tarissement ou la prise en compte des critères de morphologie bouchère dans la sélection des vaches laitières.

Les liaisons observées entre pratiques d'élevage et performances révèlent donc différentes logiques de fonctionnement d'élevage mais aussi différentes perceptions des éleveurs concernant le type génétique recherché. Les combinaisons identifiées entre pratiques et niveau de performances semblent configurer un gradient d'expression de la mixité, avec la possibilité de déplacer un curseur situant le niveau d'équilibre lait-viande exprimé par l'éleveur en fonction de ses objectifs et de ses contraintes. Le réglage de ce curseur qui pourrait constituer un atout des races mixtes souligne toutefois la difficulté inhérente à l'élaboration de programmes de sélection, recherchant cet équilibre entre aptitudes laitières et bouchères.

\section{Abstract}

Fattening practices and meat results of Simmental culled-cows: the value of a dual-purpose breed.

This study of relationships between meat results of Simmental culled-cows and fattening practices is based on slaughtering data and surveys on farms. We selected slaughtering data of culled-cows in the French Simmental breed centre area.

A first analysis allowed us to differentiate four farm groups on their meat performances (carcass weight and commercial conformation). In a second time, 25 surveys on farms enabled us to make out five groups according to farmer practices, and especially fattening practices. Maize-silage based-rations were associated with higher meat results, nevertheless pasture-based rations could be linked with interesting meat results, when associated with appropriate practices (drying up, attention paid to meat traits in animal breeding). The crossing of these two typologies (performances and practices) showed a large variability in dual-purpose situations, from zero fattening to all cows fattening with plenty of food (maize silage, concentrates). The difference between these extreme groups reached $70 \mathrm{~kg}$ of carcass weight and about one class of conformation. Among these groups, we can identify different degrees in meat character with this dualpurpose breed, according to assets or limits of livestock farming systems. These degrees showed a certain flexibility in production.

GAILLARD C., INGRAND S., BLANQUET J., DEVEVEY C., ROUX M., 2007. Relations entre pratiques de finition et performances à l'abattage de vaches de réforme Simmental Française : intérêt du caractère mixte de la race. INRA Prod. Anim., 20, 327-336. 